\title{
Renal extracapsular hypoechoic rim and kidney cortical thickness
}

\author{
Simone Brardi ${ }^{1}$, Gabriele Cevenini ${ }^{2}$, Roberto Ponchietti ${ }^{3}$, Giuseppe Romano ${ }^{4}$, Ennio Duranti ${ }^{1}$ \\ ${ }^{1}$ Hemodialysis Unit, S. Donato Hospital, Arezzo, Italy; \\ ${ }^{2}$ Department of Surgery and Bioengineering, University of Siena, Italy; \\ ${ }^{3}$ Professor of Urology, University of Siena, Italy; \\ ${ }^{4}$ Urology Unit, S. Maria della Gruccia Hospital, Montevarchi, Italy.
}

\begin{abstract}
Summary Objectives: A renal extracapsular hypoechoic rim was previously recognized and interpreted as a typical sonographic finding of renal failure. Subsequently it was hypothesized that the hypoechoic rim could be produced by a state of sodium retention and oedema caused by nephropathy but not necessarily associated with renal failure. In order to get this cleared we performed a retrospective analysis of 80 renal ultrasound examinations, carried out at our center, in 41 of which it was found a renal extracapsular hypoechoic rim.

Materials and methods: For each patient we recorded the glomerular filtration rate, the diameters in the longitudinal axis, the resistive indexes and the cortical thickness of each kidney, the possible presence and thickness of the hypoechoic rim and yet the possible coexistence of diabetes mellitus, proteinuria and clinical signs of fluid overload as peripheral oedema, distended jugular veins, ascites, increased caliber and reduced respiratory excursion of the vena cava.

Results: The F value calculated to assess the weight/influence on the hypoechoic rim of each of the variables showed as all variables, except the sex, significantly weighed on the hypoechoic rim although the greatest weight was reached by $a$ glomerular filtration rate $<60 \mathrm{ml} / \mathrm{min} / 1.73 \mathrm{~m}^{2}$ and a renal cortical thickness between 7 and $12 \mathrm{~mm}$. The hypoechoic rim was found only when cortical thickness was between 7 and 12 $\mathrm{mm}$ while it was absent if the cortical thickness was less than 7 or greater than $12 \mathrm{~mm}$. We also found numerous cases of sidedness of the hypoechoic rim.

Conclusions: It is our opinion that in case of unilateral finding of an hypoechoic rim, the association between the hypoechoic rim and the cortical thinning is consistent and therefore more accurate than the correlation between the presence of the hypoechoic rim and the reduction of the glomerular filtration rate.
\end{abstract}

KEY WORDS: Renal ultrasonography; Renal extracapsular hypoechoic rim; Cortical thinning; Kidney failure; Retrospective analysis.

Submitted 20 February 2017; Accepted 2 March 2017

\section{INTRODUCTION}

The presence of a renal extracapsular hypoechoic rim (that was called "kidney sweat" sign) (Figures 1,2) for the first time was recognized by Yassa et al. (1) in 1999 by a series of 502 ultrasound examinations, 330 of those were performed in patients with renal insufficiency. Yassa et al. (1) interpreted renal extracapsular hypoechoic rim as an ultrasound finding exclusive of patients with renal impairment because they didn't find any trace of it in any of 172 kidney ultrasound scans performed in patients who had no clinical or laboratory signs of kidney failure.

Subsequently Haddad et al. (2) by a series of only 9 patients advanced the hypothesis that the renal extracapsular hypoechoic rim could be constituted by a transudate that may occur in patients suffering from a state of sodium retention and oedema resulting from some form of nephropathy although not necessarily associated with renal impairment. However, Haddad et al. (2) observed the hypoechoic rim also in patients affected by parenchymal renal disease but without renal failure.

In order to find more evidence to settle, if possible, this different interpretations we performed a retrospective analysis of 41 renal ultrasound examinations, carried out over the last six months at our center and reporting the presence of an unilateral or bilateral renal extracapsular hypoechoic rim, in association with a retrospective analysis of 39 controls, randomly selected, among the patients who underwent renal ultrasound exam in the same six months and who didn't show any renal extracapsular hypoechoic rim.

Of these 80 patients we recorded sex and age, the actual glomerular filtration rate estimated from the serum creatinine by the Chronic Kidney Disease Epidemiology Collaboration (CKD-EPI) equation (3), the longitudinal axis diameters of each of the two kidneys, the resistive indexes of both kidneys, the cortical thickness of each of the two kidneys, the presence or not, for each kidney, of an extracapsular hypoechoic rim and, if present, its thickness, presence of diabetes mellitus, presence of proteinuria and its amount, presence of any clinical signs of fluid overload such as peripheral oedema, distended jugular veins, ascites, increased caliber and reduced respiratory excursion of the vena cava.

\section{Materials AND METHOdS}

From June 2016 to December 2016 we found at renal ultrasound examination an unilateral or bilateral renal extracapsular hypoechoic rim in 41 patients.

Subsequently we randomly selected 39 patients as control group, among who underwent renal ultrasound 
examination in the same six months and who didn't show any renal extracapsular hypoechoic rim.

All kidney nephrology ultrasound examinations were performed by Logiq S7 (GE Medical Systems Italy S.P.A. Milan, Italy) sonographic system using 3 to 5 Mhz transducers.

For every patient were recorded sex, age and glomerular filtration rate (GFR) estimated by the CKD-EPI equation (3) from serum creatinine as found during the blood test closer to the time of the ultrasound examination.

Subsequently four classes of renal function were recognized: severe renal insufficiency when the GFR was lower than $30 \mathrm{ml} / \mathrm{min} / 1.73 \mathrm{~m}^{2}$, moderate renal impairment when the GFR was equal or greater than 30 $\mathrm{ml} / \mathrm{min} / 1.73 \mathrm{~m}^{2}$ and less than $60 \mathrm{ml} / \mathrm{min} / 1.73 \mathrm{~m}^{2}$, mild renal impairment when the GFR was equal or greater than $60 \mathrm{ml} / \mathrm{min} / 1.73 \mathrm{~m}^{2}$ and less than $90 \mathrm{ml} / \mathrm{min} / 1.73$ $\mathrm{m}^{2}$ and finally normal renal function when the GFR proved equal or greater than $90 \mathrm{ml} / \mathrm{min} / 1.73 \mathrm{~m}^{2}$.

We recorded the diameters in the longitudinal axis of each kidney, the resistive indexe of each kidney by sampling intrarenal segmental and interlobar arteries and finally the cortical thickness of each kidney by measuring the portion closer to the upper pole and the lower pole of the same kidney.

Four grades of cortical thickness were recognized: severe reduction of cortical thickness when the thickness of the cortex was less than $7 \mathrm{~mm}$, moderate reduction of cortical thickness when the thickness of the cortex was greater than $7 \mathrm{~mm}$ and less than $10 \mathrm{~mm}$, mild reduction in cortical thickness when the cortical thickness was greater than $10 \mathrm{~mm}$ and less than $12 \mathrm{~mm}$ and normal cortical thickness when the thickness of the cortex was equal or greater than $12 \mathrm{~mm}$.

Of course was recorded the presence or absence, for each kidney, of a renal extracapsular hypoechoic rim and, if present, its thickness; presence of diabetes mellitus or presence of proteinuria and its amount, presence of any clinical signs of fluid overload such as peripheral oedema, distended jugular veins, ascites, increased the caliber and reduced respiratory excursion of the vena cava.

Since the perirenal spaces can collect fluid collections that may be related to acute renal disorders or related to adjacent retroperitoneal structures (4) we excluded patients with renal trauma, spontaneous rupture of renal cysts, hydronephrosis or mass lesions (5) and patients with acute pancreatitis which may cause pancreatic exudates going to gather in front pararenal spaces.

Descriptive statistics of the quantitative data was reported as the mean and standard deviation, while qualitative data as frequency and/or percentage. It was used a univariate logistic regression to evaluate the weight of each variable on the presence of the renal extracapsular hypoechoic rim, using the F statistic of Fisher. The groups were statistically compared, using the analysis of variance (ANOVA) for quantitative variables distributed in a Gaussian manner. Where it had to reject the statistical assumptions of normality, checked using the Kolmogorov-Smirnov test, was used the nonparametric Kruskal-Wallis test. When the ANOVA or Kruskal-Wallis test provided statistically significant differences between the test groups, we used, respectively, the post-hoc test Bonferroni or Dunn for pairwise comparisons. The comparison between groups for qualitative variables was performed with Fisher's exact test for 2 x 2 contingency tables, otherwise, with chi-square test for the greater dimension tables. Finally, the correlations between quantitative variables were performed by calculating the Pearson correlation coefficient and evaluating statistical significance. For all statistical analyzes, performed with the SPSS software package version 10, it was chosen a significance level of $95 \%$ and thus were considered statistically significant $\mathrm{p}<0.05$ values.

\section{Results}

A renal extracapsular bilateral hypoechoic rim was observed in 21 patients (18 males and 3 females), mean age $78.1 \pm 10.1$ years with an average renal cortical thickness of $0.52 \pm 0.17 \mathrm{~cm}$, a minimum thickness of $0.24 \mathrm{~cm}$ and a maximum thickness of $0.94 \mathrm{~cm}$; these 21 patients had a mean glomerular filtration rate of $34.5 \pm$ $12.2 \mathrm{ml} / \mathrm{min} / 1.73 \mathrm{~m}^{2}$ and a renal cortical thickness greater of $7 \mathrm{~mm}$ and less than $10 \mathrm{~mm}$ in $31 \%$ of the cases and a renal cortical thickness greater than $10 \mathrm{~mm}$ and less than $12 \mathrm{~mm}$ in the remaining 69\%.

The mean diameter in longitudinal axis was $98.3 \pm 11.6$ $\mathrm{mm}$ with a maximum of $130 \mathrm{~mm}$ and a minimum of 71 $\mathrm{mm}$. The average of resistive indexes were $0.73 \pm 0.06$; proteinuria was found in $62 \%$ of patients (13 of 21 patients) and the average amount was $0.33 \pm 0.59 \mathrm{~g} / 24 \mathrm{~h}$. Finally signs of fluid overload were observed in 19\% of patients while diabetes was present in 33\% of the same patients.

Instead a renal extracapsular unilateral hypoechoic rim was found in 20 patients (10 males and 10 females), mean age $81.1 \pm 7.2$ years, with an average renal cortical thickness of $0.27 \pm 0.29 \mathrm{~cm}$, a minimum of $0.24 \mathrm{~cm}$ and a maximum of $0.79 \mathrm{~cm}$; these patients had a mean glomerular filtration rate of $34.7 \pm 12 \mathrm{ml} / \mathrm{min} / 1.73 \mathrm{~m}^{2}$ while cortical thickness was greater than $7 \mathrm{~mm}$ and less than $10 \mathrm{~mm}$ in $55 \%$ of kidneys with unilateral hypoechoic rim and greater than $10 \mathrm{~mm}$ and less than $12 \mathrm{~mm}$ in the remaining $45 \%$ of the same kidneys, while in the other 20 kidneys without extracapsular hypoechoic rim was found a renal cortical thickness smaller than $7 \mathrm{~mm}$ in a percentage of $55 \%$ and a cortical thickness equal or greater than $12 \mathrm{~mm}$ in the remaining $45 \%$. The average diameter of the kidneys with an unilateral hypoechoic rim in the longitudinal axis was $90.6 \pm 17.8 \mathrm{~mm}$ with a maximum of $126 \mathrm{~mm}$ and a minimum $55 \mathrm{~mm}$. The mean resistive index was $0.76 \pm 0.07$; proteinuria was found in $63 \%$ of patients and the average amount was $0.21 \pm$ $0.35 \mathrm{~g} / 24 \mathrm{~h}$. Finally signs of fluid overload were found in $10 \%$ of patients with unilateral hypoechoic rim while diabetes was found in a 30\% of these same patients.

The patients without both unilateral or bilateral extracapsular hypoechoic rim were 39, 22 males and 17 females, with a mean age of $57.5 \pm 15.1$ years while the glomerular filtration rate was $77 \pm 22.8 \mathrm{ml} / \mathrm{min} / 1.73 \mathrm{~m}^{2}$. The mean diameter in the longitudinal axis was $108.1 \pm$ $16.6 \mathrm{~mm}$ with a maximum of $143 \mathrm{~mm}$ and a minimum of $92 \mathrm{~mm}$; the average resistive index was $0.63 \pm 0.06$. The cortical thickness was less than $7 \mathrm{~mm}$ in $18 \%$ of the total of the kidneys and equal or greater than $12 \mathrm{~mm}$ in 
Table 1.

Weight/influence of the variables on the hypoechoic rim (unilateral and/or bilateral).

\begin{tabular}{|lcc|}
\hline Variables & $\begin{array}{c}\text { Score } \\
\text { (Fisher F) }\end{array}$ & $\begin{array}{c}\text { Statistical } \\
\text { significance (p-value) }\end{array}$ \\
\hline Age (years) & 28.219 & .000 \\
\hline Sex & 2.262 & .133 \\
\hline GFR (ml/min/1.73 $\left.{ }^{2}\right)$ & 37.556 & .000 \\
\hline Right kidney diameter $(\mathrm{mm})$ & 7.305 & .007 \\
\hline Left kidney diameter $(\mathrm{mm})$ & 13.262 & .000 \\
\hline Right kidney cortical thickness $(\mathrm{mm})$ & 42.134 & .000 \\
\hline Left kidney cortical thickness $(\mathrm{mm})$ & 50.699 & .000 \\
\hline Right kidney resistive indexes & 28.916 & .000 \\
\hline Left kidney resistive indexes & 26.900 & .000 \\
\hline Proteinuria (gr/24h) & 4.590 & .032 \\
\hline Right kidney hypoechoic rim thickness $(\mathrm{cm})$ & 32.259 & .000 \\
\hline Left kidney hypoechoic rim thickness $(\mathrm{cm})$ & 41.944 & .000 \\
\hline Clinical findings of fluid overload & 5.628 & .018 \\
\hline Diabetes mellitus & 13.867 & .000 \\
\hline Right kidney cortical thickness $>7<12(\mathrm{~mm})$ & 45.490 & .000 \\
\hline Left kidney cortical thickness $>7<12(\mathrm{~mm})$ & 49.568 & .000 \\
\hline GFR $<60$ (ml/min/1.73 $\left.{ }^{2}\right)$ & 49.568 & .000 \\
\hline
\end{tabular}

the remaining $82 \%$. Signs of fluid overload and diabetes were not found in anyone of the patients.

The F value calculated to assess the weight on the presence of unilateral or bilateral renal extracapsular hypoechoic rim of each of the variables taken into account (age, sex, glomerular filtration rate, diameter of each kidney, cortical thickness of each kidney, resistive index of each kidney, presence of proteinuria, presence of signs of fluid overload, presence of diabetes mellitus) allowed us to demonstrate that all the variables except gender (which showed a p-value $>0.05$ ) weighed significantly on yhe presence of an hypoechoic rim (Table 1).

However the greater weight on the presence of the renal extracapsular hypoechoic rim was equally reached by both the presence of a glomerular filtration rate lower than 60 $\mathrm{ml} / \mathrm{min} / 1.73 \mathrm{~m}^{2}$ and by the presence of a cortical thickness greater than 7 and less than $12 \mathrm{~mm}$ (Table 1).

Furthermore it was found in a statistically significant manner that the renal extracapsular hypoechoic rim was present only when cortical thickness was greater than 7 $\mathrm{mm}$ and less than $10 \mathrm{~mm}$ or greater than 10 and less than $12 \mathrm{~mm}$ while it was not found any extracapsular hypoechoic rim when the cortical thickness was less than $7 \mathrm{~mm}$ or greater than $12 \mathrm{~mm}$.

It was not found any correlation between severe reduction of the glomerular filtration rate (i.e. $<30 \mathrm{ml} / \mathrm{min} / 1.73 \mathrm{~m}^{2}$ ) or moderate reduction of the glomerular filtration rate (i.e. $\geq 30$ and $<60 \mathrm{ml} / \mathrm{min} / 1.73 \mathrm{~m}^{2}$ ) and the respective mean thickness of the extracapsular hypoechoic rim.

It was also confirmed the presence of a statistically significant correlation between the presence of signs of fluid overload and the coexistence of renal extracapsular hypoechoic rim although this association was found in only 6 of the 41 patients with an unilateral or bilateral renal extracapsular hypoechoic rim that is the $15 \%$ of the cases.

A similar statistically significant correlation was found between the presence of hypoechoic rim and the coexistence of diabetes mellitus or proteinuria.

\section{Discussion}

The kidney is encased by the anterior and posterior pararenal fascia, also known as Gerota's fascia, forming the perirenal space. Between the anterior pararenal fascia and the posterior peritoneum there is the anterior pararenal space while between the posterior pararenal fascia and the transversalis fascia there is the posterior pararenal space (6-8). The fascia cannot be recognized by sonography so the perirenal and pararenal spaces appear as a single compartment filled with fat $(6,7)$.

The fat is variable in amount and is usually hyperechoic but occasionally may become hypoechoic, when the water content of the fat tissue is low $(6,9)$, so appearing as a hypoechoic rim (Figures 1,2$)$ that mimics a perirenal fluid $(6,10,11)$.

Figure 1.

Renal extracapsular hypoechoic rim (arrows).

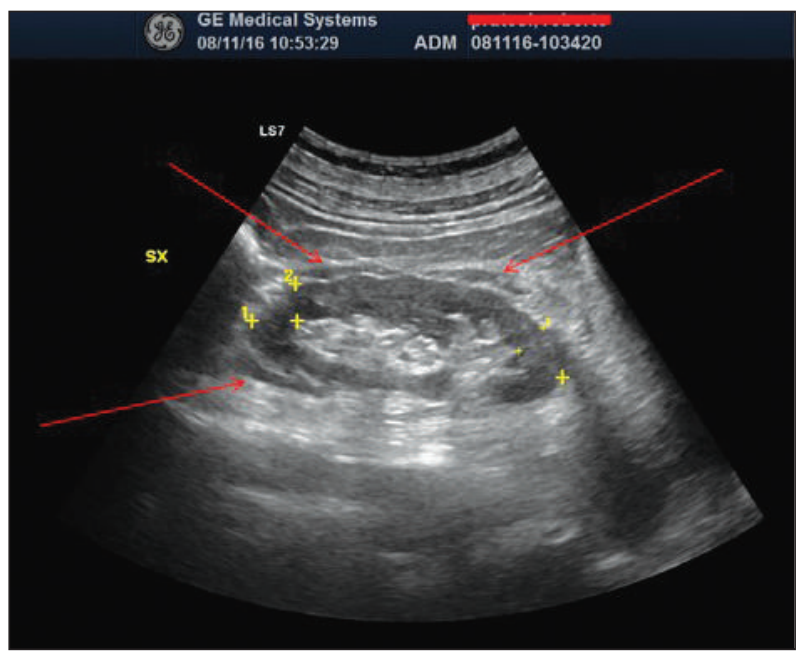

Figure 2.

Renal extracapsular hypoechoic rim (arrow).

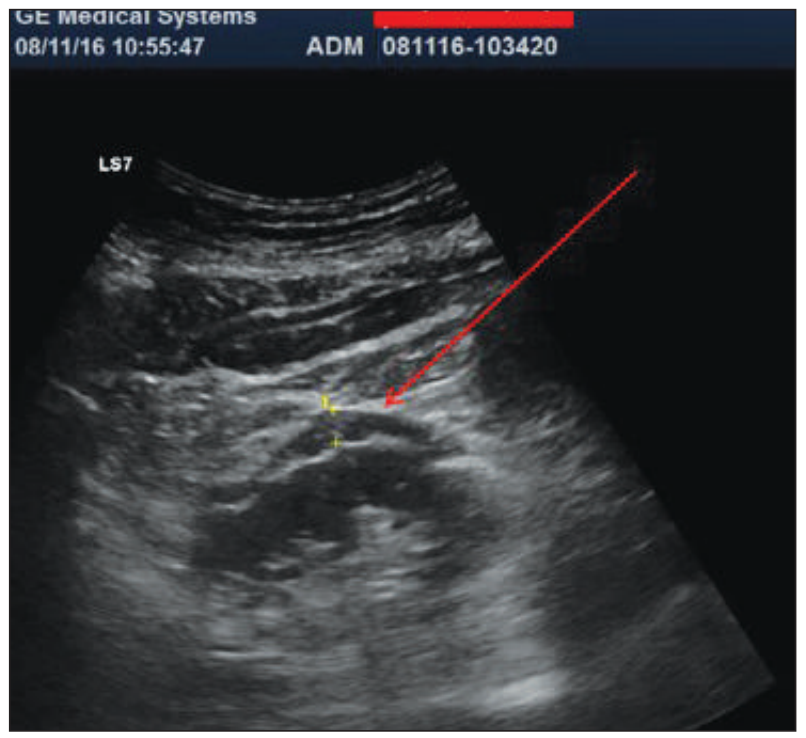


Moreover the ultrasound appearance of the kidneys under chronic renal failure $(6,12,13)$ it is characterized by a reduction in renal size, thinning of the parenchyma (especially of the cortex) and increased echogenicity of the same cortex. In these cases when cortical thickness cannot be determined with precision because the medullary pyramids are not visible, thinning of the cortex can be appreciated as thinning of the entire parenchyma (6).

With respect to normal cortical thickness, although there are limited data, a cutoff of $12 \mathrm{~mm}$ can be considered appropriate $(6,14)$ while a value of less than $7 \mathrm{~mm}$ is probably abnormal (6). Among the all characteristics of cortical atrophy, the most reliable is just the cortical thinning (6) since the renal size varies with body size and increased echogenicity is a subjective determination that may be affected by artifacts (6). Consequently echogenicity should not be taken into account among the parameters which can weigh on the hypoechoic rim.

More in detail the renal cortex is the subcapsular portion of the kidney composed mainly of glomeruli and convoluted tubules, extending in columns between the pyramids that constitute the renal medulla. Renal ultrafiltration, which occurs in the glomeruli, takes place therefore in the cortex of the kidney. Given the above, we note that, consistently with the findings of Yassa et al. (1) and Haddad et al. (2), also our study shows a statistically significant close correlation between the presence of the hypoechoic rim and a reduced glomerular filtration rate below $60 \mathrm{ml} / \mathrm{min} / 1.73 \mathrm{~m}^{2}$, as well as a correlation between the presence of the hypoechoic rim and the coexistence of fluid overload although this latter correlation, in the light of the limited number of the cases of fluid overload (which were only the 15\% of all patients with unilateral or bilateral hypoechoic rim), certainly weighs less in statistical terms on hypoechoic rim if compared to the presence of above-mentioned renal insufficiency as shown by the specific statistical analysis (Table 1). It is also widely known as the ascites is a common cause of widening of the space between the right kidney and the liver (6).

Indeed, quite peculiar of this retrospective analysis, it is the close correlation that was found between the presence of the hypoechoic rim and a thinning of the renal cortex between 7 and $12 \mathrm{~mm}$ so that the reduction in cortical thickness weighs on the hypoechoic rim as the presence of a glomerular filtration rate less than 60 $\mathrm{ml} / \mathrm{min} / 1.73 \mathrm{~m}^{2}$.

Furthermore we found in our series numerous cases of unilaterality of the hypoechoic rim equal to the $49 \%$ of all patients that showed the hypoechoic rim, quite differently from what found by Yassa et al. (1) who observed a bilateral hypoechoic rim in all cases but accordingly to Haddad et al. (2) and Orofino et al. (16) who reported cases unilaterality of the hypoechoic rim.

The association between the hypoechoic rim and a reduction in cortical thickness, excluding conditions of excessive reduction of cortical thickness (as when below to $7 \mathrm{~mm}$ or absent ) or ortical thickness $\geq 12 \mathrm{~mm}$ is consistent and in the frequent cases where the presence of the hypoechoic rim is inilateral this associaytion is undoubtedly more accurate when compared to the sim- plest correlation between the presence of the hypoechoic rim and reduction of glomerular filtration rate below 60 $\mathrm{ml} / \mathrm{min} / 1.73 \mathrm{~m}^{2}$.

Although it is true that a reduction in the glomerular filtration rate below $60 \mathrm{ml} / \mathrm{min} / 1.73 \mathrm{~m}^{2}$ it is often accompanied by a cortical thinning it is equally true that in many cases the kidney damage (of all kinds) may be unilateral or otherwise more expressed in one of the two kidneys involving a different cortical thickness between a kidney and the other (as observed in our series, in a kidney cortical thickness may be even normal and in the other completely lost i.e less than $7 \mathrm{~mm}$ ) while renal function (as assessed by estimated glomerular filtration rate which is calculated from serum creatinine) it is given by the sum of the glomerular filtration rate of each kidney, one of which can compensate completely or in part to the failure of the other.

Finally our series showed that the hypoechoic rim was absent when the cortical thickness was completely lost i.e less than $7 \mathrm{~mm}$, as is the case of end-stage kidneys, in fact the hypoechoic rim, likewise the thinning of the renal cortex, may be a finding of kidney damage that will disappear when renal function will become negligible or lost and the cortical thickness, at the same time, will shrink.

\section{Conclusions}

It is our opinion that, except in cases of fluid overload, the hypoechoic rim, accordingly to the thinning of renal cortex to which it is very well correlated, can be regarded as an expression of damage, probably chronic, of the single kidney in which it is found and therefore it is associated, of course, with a reduction of renal function.

To explain the absence of the hypoechoic rim in case of complete loss of cortical thickness (i.e. $<7 \mathrm{~mm}$ ), we can hypothesize that the presence of the hypoechoic rim may be associated only with an initial phase of renal injury that still has not hesitated in a complete functional loss of the single kidney.

It is clear, however, that further trials are needed to confirm this hypothesis.

\section{References}

1. Yassa NA, Peng M, Ralls PW. Perirenal lucency (kidney sweat'): a new sign of renal failure. AJR Am J Roentgenol. 1999; 173:1075-1077.

2. Haddad MC, Medawar WA, Hawary MM, et al. Perirenal fluid in renal parenchymal medical disease (floating kidney'): clinical significance and sonographic grading. Clinical radiology. 2001; 56:979-983.

3. Levey AS, Stevens LA, Schmid CH, et al. A new equation to estimate glomerular filtration rate. Ann Intern Med. 2009; 150:604-12.

4. Haddad MC, Hawary MM, Khoury NJ, et al. Radiology of perinephric fluid collections. Clinical Radiology. 2002; 57:339-346.

5. Balci NC, Akun E, Erturk M, et al. Renal-related perinephric fluid collections: MRI findings. Magnetic Resonance Imaging. 2005; 23:679-684.

6. O'Neill W. C. Atlas of renal ultrasonography. 2001 W.B. Saunders Company. 
7. Belli A-M, Joseph AEA. The renal rind sign: a new ultrasound indication of inflammatory disease in the abdomen. $\mathrm{Br} \mathrm{J}$ Radiol. 1988; 61:806.

8. Chen J-J, Changchien C-S, Kuo C-H. Causes of increasing width of right anterior extrarenal space seen in ultrasonographic examinations. J Clin Ultrasound. 1995; 23:287.

9. Behan M, Kazam E. The echographic characteristics of fatty tissues and tumors. Radiology. 1978; 129:143.

10. Brammer HM, Smith WS, Lubbers PR. septated hypoechoic perirenal fat on sonograms: a pitfall in renal ultrasonography. $J$ Ultrasound MEd. 1992; 11:361.

11. Heinz-Peer G, Oettl C, Mayer G, et al. Hypoechoic perirenal fat in renal transplant recipients. Radiology. 1994; 193:717.
12. Paivansalo M, Huttunen K, Suramo I. Ultrasono graphic findings in renal parenchymal diseases. Scan J Urol Nephrol. 1985; 19:119.

13. Hricak H, Cruz C, Romanski R, et al. Renal parenchymal disease: sonographic-histologic correlation. Radiology. 1982; 144:141.

14. Emamian SA, Nielsen MB, Pedersen JF, et al. Kidney dimensions at sonography: correlation with age, sex and habitus in 665 adult volunteers. AJR. 1993; 160:83.

15. Raj DSC, Hoisala, Somiah S, et al. Quantitation of change in the medullary compartment in renal allograft by ultrasound. J Clin Ultrasound. 1997; 25:265.

16. Orofino L, Herrero A, Quereda C, et al. Perirenal subcapsular fluid collection in a patient with membranous nephropathy and renal vein thrombosis. J Urol. 1986; 136:1287-1289.

\section{Correspondence}

Simone Brardi, MD

sibrardi@gmail.com

Ennio Duranti, MD

ennio.duranti@libero.it

Hemodialysis Unit, S. Donato Hospital, Arezzo, Italy

Gabriele Cevenini, MD

cevenini@unisi.it

Department of Surgery and Bioengineering, University of Siena, Siena, Italy

Roberto Ponchietti, MD

ponchietti@unisi.it

Professor of Urology, University of Siena, Siena, Italy

Giuseppe Romano, MD

giuseppe.romano@usl8.toscana.it

Urology Unit, S. Maria della Gruccia Hospital, Montevarchi, Italy 